\title{
Interaction of two non-coalescing bubbles rising in a non-isothermal self-rewetting fluid
}

\author{
Mounika Balla ${ }^{a}$, Manoj Kumar Tripathi ${ }^{b}$, Omar K. Matar ${ }^{c}$ and Kirti Chandra \\ $\mathrm{Sahu}^{a 1}$ \\ ${ }^{a}$ Department of Chemical Engineering, Indian Institute of Technology Hyderabad, \\ Sangareddy 502 285, Telangana, India \\ ${ }^{b}$ Indian Institute of Science Education and Research Bhopal 462 066, Madhya Pradesh, \\ India \\ ${ }^{c}$ Department of Chemical Engineering, Imperial College London, London SW7 2AZ, UK
}

\begin{abstract}
The attractive and repulsive behaviours of a pair of initially spherical gas bubbles rising side-by-side in a channel with non-uniformly heated walls containing a self-rewetting liquid are investigated numerically. The surface tension of a self-rewetting fluid exhibits a parabolic temperature dependence with a welldefined minimum, as opposed to linear (common) fluids whose surface tension decreases almost linearly with the increasing temperature. It is found that, for low Reynolds numbers, while in an isothermal medium, two gas bubbles display a repulsive behaviour, they attract in non-isothermal systems. The bubbles in the self-rewetting fluid undergo a plastic collision and show a 'squeezing and relaxing' behaviour, whereas they attract and then bounce in the linear fluid. A regime map demarcating the repulsive and attractive behaviours for a selfrewetting fluid is plotted in the Weber number $(W e)$ and the dimensionless linear component of the surface tension gradient $\left(M_{1}\right)$ space. It is found that the bubbles in the self-rewetting fluid remain spherical even for high Weber numbers while they deform considerably in the case of the linear fluid indicating that the attractive behaviour of the bubbles in the self-rewetting fluid is due to the lift force generated by the thermocapillary stresses and not due to the de-
\end{abstract}

\footnotetext{
${ }^{1}$ Email: ksahu@che.iith.ac.in
} 
formation. The mechanism underlying the observed phenomenon is elucidated by studying the drag and lift forces acting on the bubbles, their orientations, and the flow field around them.

Keywords: Bubble dynamics, Marangoni stresses, Self-rewetting fluid, Non-isothermal flow, Numerical simulation, Collision

\section{Introduction}

The interaction of bubbles rising together in liquid is relevant in many industrial applications, e.g. bubble column reactors and heat exchangers [1, 2], and also in natural phenomena [3, 4]. Gaseous bubbles are commonly used in industrial applications to improve heat and mass transfer, and which depends primarily on interaction and spatial distribution of the bubbles. Thus, several researchers have investigated the motion of gaseous bubbles in a liquid medium in isothermal (e.g. Refs. [5] ditions. In this context, two bubbles rising side by side have been considered as a model problem for investigating the interactions between them in both isothermal and non-isothermal systems.

The previous investigations conducted on the interactions and trajectories of a pair of side-by-side rising spherical bubbles in liquids due to buoyancy under isothermal condition reveal that two spherical bubbles/drops, which in Stokes flow would rise with constant separation, repel each other at low Reynolds numbers and attract each other at high Reynolds numbers (see e.g. Ref. [5, 6]). Furthermore, 7] showed that the rise dynamics of two gaseous bubbles rising side-by-side is planar at low Reynolds numbers. An extensive review of two bubbles rising side-by-side in isothermal systems can also be found in Refs. [7. 8.

In non-isothermal systems, in addition to buoyancy, the thermocapillary stresses resulting from the surface tension gradient in the vicinity of the gasliquid interface also play an important role in the dynamics of the bubbles. The surface tension, $\left(\sigma_{d}\right)$ of common fluids (hereafter referred to as 'linear' fluids), 
such as water and various oils usually decreases almost linearly with increasing temperature $\left(T_{d}\right)$. Young et al. [12] showed that a small spherical bubble migrates against buoyancy in the downward direction when the bottom of a container holding a linear fluid is significantly hotter than the top. Under the influence of both buoyancy and thermocapillary forces, they also established a theoretical model to predict the terminal velocity of the bubble. Since then, several researchers have investigated the motion of a single bubble (e.g. Refs. [13 16]) and multiple bubbles (e.g. Refs. [10, 11]) in linear fluids due to an imposed temperature gradient. A few researchers have also studied a pair of bubbles/drops rising in linear fluids in non-isothermal systems. Wei and Subramanian [17] studied the migration of a pair of spherical gaseous bubbles in a linear fluid subjected to a combined action of gravity and a downward temperature gradient in the Stokes flow limit. Lavrenteva et al. [18] and Frolovskaya et al. [19] investigated the interaction of thermal wakes in the case of in-line deformed drops rising in a linear fluid and extended the study of Wei and Subramanian [17] to the high Péclet number regime.

In contrast to linear fluids, the surface tension of non-azeotropic, high carbon alcohol solutions, exhibits a quasi-parabolic temperature dependency. Increasing the alcohol concentration in the solution has been found to increase the parabolicity of the $\sigma_{d}-T_{d}$ curves [20 22]. Subsequently, Abe et al. [23] termed these fluids as 'self-rewetting' fluids. Due to the unusual behaviour of the surface tension, several researchers investigated the spreading dynamics of self-rewetting sessile drops on a heated surface [24, 25]. It was found that a sessile drop placed at the location of the minimum surface tension exhibits a super-spreading behaviour [24].

Several researchers have also examined the dynamics of a single bubble in self-rewetting fluids. Tripathi et al. [26] and Balla et al. [27] investigated the motion of a gaseous bubble in a container maintained at a linearly increasing temperature field and filled with a self-rewetting fluid. The gaseous bubble was shown to exhibit complex dynamics while moving in the channel due to the combined action of buoyancy and thermocapillary stresses. Duffy et al. 228 the- 
oretically investigated the dynamics of a long bubble in a non-uniformly heated tube containing a self-rewetting fluid under the action of imposed axial flow and gravitational effect. The dynamics of an oil droplet in a stratified liquid of binary mixtures exhibiting a parabolic surface tension gradient was investigated in Refs. 29 31. They observed self-propelling and bouncing behaviours of the oil droplet due to the Marangoni stresses in the vertical direction.

As the above literature review shows, the dynamics of two bubbles/drops rising side-by-side in a self-rewetting fluid has not yet been studied, despite the fact that these fluids are preferred in many practical applications due to their significantly higher critical heat fluxes and also due to their relevance in space applications [32. In the present study, we investigate the interaction of two non-coalescing gas bubbles rising in a non-isothermal self-rewetting fluid, which is contrasted with the dynamics observed in the cases of linear and isothermal systems. The bubbles are considered non-coalescing because of their small size and less contact time for the coalescence to occur. A two-dimensional geometry is also considered in view of the finding reported by Tripathi et al. [7] on the planar motion of the bubbles at low Reynolds number. We found that while in an isothermal system, two bubbles repel each other at low Reynolds numbers, they attract each other in a non-isothermal system. Our findings suggest that interaction of the bubbles in a self-rewetting fluid is significantly different from that observed in isothermal and linear systems. A regime map is plotted demarcating the attractive and repulsive behaviours in the Weber number $(W e)$ and the dimensionless linear component of the surface tension gradient with temperature $\left(M_{1}\right)$ space. The mechanism for this behaviour is investigated by analysing the lift force between the bubbles and the flow field.

The rest of the paper is organised as follows. The problem is formulated and governing equations are described in Section 2. The present numerical method and the validation of the solver are also presented in this section. The numerical results and the underlying physics are discussed in Section 3 . Finally, concluding remarks are given in Section 4 . 
(a)

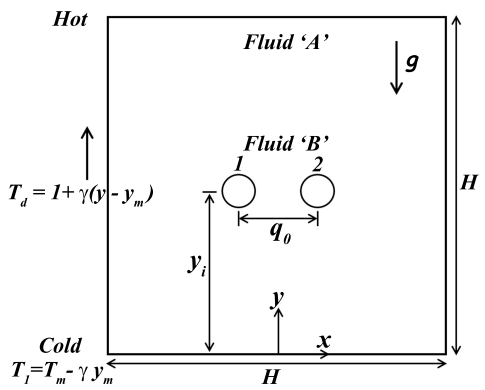

(b)

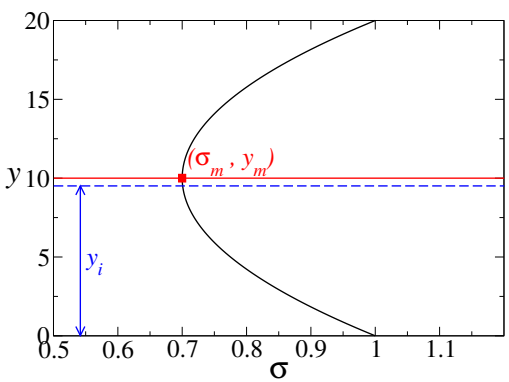

Figure 1: (a) Schematic diagram showing the initial configuration of two gas bubbles (fluid $B$ ) rising side-by-side inside a self-rewetting fluid (fluid $A$ ) starting from $y=y_{i}$; initially separated by a distance $q_{0}$. (b) The surface tension, $\sigma$, profile for $M_{1}=0.6$ and $M_{2}=0.3$ (Eq. 1); $\sigma$ exhibits a minimum, $\sigma_{m}$ at $y=y_{m}$.

\section{Formulation}

We studied numerically the motion of two non-coalescing gas bubbles (fluid $B$ ) of the same initial radius $R$ inside a two-dimensional channel (with $H=$ $20 R$ ) filled with a self-rewetting fluid (fluid $A$ ). The fluids are assumed to be incompressible and Newtonian. The viscosity and density of fluids $A$ and $B$ are $\left(\mu_{A}, \rho_{A}\right)$ and $\left(\mu_{B}, \rho_{B}\right)$, respectively. A Cartesian coordinate system $(x, y)$ is used, such that the acceleration due to gravity, $g$ acts in the negative $y$ direction, as shown in Fig. 17. The initial locations of bubbles '1' and '2' are $\left(-q_{0} / 2, y_{i}\right)$ and $\left(q_{0} / 2, y_{i}\right)$, respectively, where $q_{0}$ is the initial separation distance between the bubbles. The rigid and impermeable walls with a linear temperature gradient, $\gamma$, are located at $x= \pm H / 2$.

To perform non-dimensionalisation, $R, V\left(\equiv \beta_{1} \gamma R / \mu_{A}\right), t_{s}\left(\equiv \mu_{A} / \beta_{1} \gamma\right)$ are used as the reference length, velocity and time scales, respectively. The properties of fluid $A$ and $\sigma_{0}$ (the surface tension at temperature $T_{1}$ ) are used to nondimensionalise the respective variables. The dimensionless temperature, $T$ is given by $\left(T_{d}-T_{1}\right) /\left(T_{m}-T_{1}\right)$, where $T_{m}$ and $T_{1}$ are the values of the temperature $\left(T_{d}\right)$ at the location of the minimum surface tension $\left(y=y_{m}\right)$ and the bottom of the domain $(y=0)$, respectively. The dimensionless surface tension, 
$\sigma$ as a function of $T$ is given by

$$
\sigma=1-M_{1} T+M_{2} T^{2}
$$

where $M_{1}=\beta_{1}\left(T_{m}-T_{1}\right) / \sigma_{0}$ and $M_{2}=\beta_{2}\left(T_{m}-T_{1}\right)^{2} / \sigma_{0}$, wherein $\beta_{1}=$ $-d \sigma_{d} /\left.d T_{d}\right|_{T_{1}}$ and $\beta_{2}=\left(d^{2} \sigma_{d} / d T_{d}^{2}\right) /\left.2\right|_{T_{1}}$. A typical surface tension profile for $M_{1}=0.6$ and $M_{2}=0.3$ is shown in Fig. 1(b). We always set $M_{2}=M_{1} / 2$ to ensure that the location of the minimum surface tension at the middle of the computational domain, i.e. $y_{m}=10$.

The dimensionless governing equations are given by

$$
\begin{gathered}
\nabla \cdot \mathbf{u}=0 \\
\rho\left[\frac{\partial \mathbf{u}}{\partial t}+\mathbf{u} \cdot \nabla \mathbf{u}\right]=-\nabla p+\frac{1}{R e} \nabla \cdot\left[\mu\left(\nabla \mathbf{u}+\nabla \mathbf{u}^{T}\right)\right]+\frac{\delta}{W e}\left(\sigma \kappa \mathbf{n}+\nabla_{s} \sigma\right)-\frac{\rho \vec{e}_{y}}{F r} \\
\frac{\partial T}{\partial t}+\mathbf{u} \cdot \nabla T=\frac{1}{M a} \nabla \cdot(\alpha \nabla T),
\end{gathered}
$$

where $\mathbf{u}, p$ and $T$ denote the velocity, pressure, and temperature fields, respectively; $t$ represents time; $\delta$ is a delta distribution that is zero everywhere except at the interface; $\kappa=\nabla \cdot \mathbf{n}$ is the curvature, $\mathbf{n}$ is the unit normal to the interface pointing towards fluid $A ; \vec{e}_{y}$ represents the unit vector in the vertically upward direction; $\nabla_{s}$ represents the surface gradient operator that is calculated directly using the height functions in the volume of fluid (VoF) framework. The normal stress $(\sigma \kappa \mathbf{n} / W e)$ and tangential (Marangoni) stress $\left(\nabla_{s} \sigma / W e\right)$ at the gas-liquid interface is included in Eq. (3) based on the continuum surface force formulation. The calculation procedure of the Marangoni term is described in section 1 of the supplementary information.

The various dimensionless numbers are the Reynolds number $\left(R e \equiv \rho_{A} V R / \mu_{A}\right)$, the Froude number $(F r \equiv V / \sqrt{g R})$, the Weber number $\left(W e \equiv \rho_{A} R V^{2} / \sigma_{0}\right)$ and the Marangoni number $\left(M a \equiv V R / \alpha_{A}(\equiv \operatorname{Re} \operatorname{Pr})\right)$, where $\operatorname{Pr}\left(\equiv \mu_{A} / \rho_{A} \alpha_{A}\right)$ is the Prandtl number. The dimensionless temperature gradient, $\Gamma$ is given by $\gamma R /\left(T_{m}-T_{1}\right)$.

The dimensionless viscosity $(\mu)$, density $(\rho)$ and thermal diffusivity $(\alpha)$ are 
given by 26 ]

$$
\begin{gathered}
\mu=c e^{-T}+(1-c) \mu_{r}\left(1+T^{3 / 2}\right), \\
\rho=c+\rho_{r}(1-c), \\
\alpha=c+\alpha_{r}(1-c),
\end{gathered}
$$

where $\mu_{r} \equiv \mu_{B} / \mu_{A}, \rho_{r} \equiv \rho_{B} / \rho_{A}$ and $\alpha_{r} \equiv \alpha_{B} / \alpha_{A}$ are viscosity, density and thermal diffusivity, respectively. Here, $c$ is the volume fraction of the liquid phase, such that $c=1$ and 0 for fluids $A$ and $B$, respectively. In our numerical simulations, the interfaces of the two bubbles are associated with two different colour functions in the VoF framework. This causes the bubbles to maintain their interfaces even if they share a common computational cell. The current solver employs the conservative method proposed by Weymouth and Yue [33] for the advection of color functions and does not use any other artificial condition to circumvent the problem of crossover of the interfaces. It should be noted that this condition is only physically true if the bubbles are considered a priori to be non-coalescing.

Since the current formulation and numerical method are the same as those given in Refs. [16, 27, we refer the reader to our previous studies for details, but briefly highlight this here for the sake of completeness. Note that 7 has also shown that the bubble rise dynamics is two-dimensional in the parameter range considered in the present study. The tangential gradient of surface tension force (Marangoni force) is implemented Tripathi and Sahu [16] in an opensource finite-volume VoF based multiphase flow solver, Basilisk [34, 35]. An adaptive mesh refinement near the interfacial and regions with vortical flow is used. No-slip and no penetration boundary conditions, a temperature field, $T=1+\Gamma\left(y-y_{m}\right)$ is imposed at all the side walls. At the top and bottom of the computational domain, the Neumann boundary conditions are used for temperature and velocity components. The solver has been validated extensively in [16, 27. A grid convergence (see Fig. 1 in the supplementary information) is performed to select the optimal mesh (with the smallest grid size, $\Delta=0.019$ ) used in this study. 


\section{Results and discussion}

We begin the presentation of our results by examining the temporal variations of the centre of gravity of bubble $1\left(y_{C G}\right)$ (see Fig. 2(a)) and the normalised separation distance between the bubbles $\left(q / q_{0}\right)$ (see Fig. 2(b)) rising in the isothermal $\left(M_{1}=M_{2}=0\right)$, linear $\left(M_{1}=0.4, M_{2}=0\right)$ and self-rewetting $\left(M_{1}=0.4, M_{2}=0.2\right)$ fluids. Here, $q_{0}=3$ and the rest of the parameters are $\Gamma=0.1, R e=10, W e=0.05, F r=50, \operatorname{Pr}=0.7, \mu_{r}=10^{-2}, \rho_{r}=10^{-3}$, $\alpha_{r}=0.04$ and $y_{i}=9.5$ (hereafter termed as 'base' parameters). The dimensionless parameters considered in the present study have been justified below.

In a gas-liquid system, the ratios of viscosity, density, and thermal diffusivity are of the same order as those considered in the present study (i.e. $\mu_{r}=10^{-2}$, $\rho_{r}=10^{-3}$ and $\left.\alpha_{r}=0.04\right)$. The value of the Prandtl number for water is about 0.71 , so it is reasonable to take $\operatorname{Pr}=0.7$ as it is defined based on fluid 'A' (i.e., the liquid phase). The velocity scale used in the present study is $\beta_{1} \gamma R / \mu_{A}$, so $F r=50$ implies that the Marangoni flow based velocity is 50 times the neutral buoyant velocity $(\sqrt{g R})$. As we investigate the thermo-capillary driven flow of a millimetre size bubble, one can also easily achieve the value of Reynolds number $(R e=10)$ and the range of the Weber number considered in the present study. Recently, Mamalis et al. [36] have measured the surface tension of solutions of water +1 -butanol $5 \%$ volume and water +1 -pentanol $2 \%$ volume and showed that surface tensions of these fluids exhibit parabolic dependencies with temperature (see Figure 4 of Ref. [36]). In the self-rewetting case, we have taken $M_{2}=M_{1} / 2$ to fix the location of minimum surface tension at $y=10$. Moreover, the parameter values considered in the present study are consistent with our previous studies [26, 27, where we investigate the thermo-capillary migration a gaseous bubble in a self-rewetting fluid. We found that the variation of the centre of gravity of bubble 2 is found to be the mirror image of that of bubble 1 about $x=0$ for all the sets of parameters considered in the present study. It can be seen in Fig. 2(a) that in both the isothermal system and nonisothermal linear fluid system, the bubble exhibits an acceleration phase and 
reaches a terminal speed. In the case of linear fluid, the acceleration period is much shorter and the terminal velocity is higher as compared to the isothermal case. This is due to the thermocapillary stresses pushing linear fluid A towards the cold region and thus increasing the upward movement of the bubbles in the case of linear fluid. In the self-rewetting fluid, the dynamics is complex. In this case, the bubbles accelerate for a short duration as $y_{i}<y_{m}$ and achieve a constant speed at the intermediate time (for $y<y_{m}$, where the thermocapillary and buoyancy forces act in the same direction). After crossing the location of the minimum surface tension $\left(y_{m}\right)$, the thermocapillary and buoyancy forces act in the opposite directions and the bubbles exhibit a decelerating phase. At this stage, they touch each other (see Fig. 2(b) at $t \approx 16$ for the self-rewetting fluid) and experience a combined buoyancy force that dominates the thermocapillary force acting in the downward direction leading to a constant upward speed at $t>16$. As the bubbles rise in the upward direction to a hotter region $\left(y>y_{m}\right)$, the thermocapillary force continues to increase because of the self-rewetting nature of the surrounding fluid, and at $t \approx 40$, the downward thermocapillary force becomes larger than the upward buoyancy force. Thus, at later stage, the bubbles reverse the direction of motion (see the inset in Fig. 2(a)). The trajectories of bubbles 1 and 2 in the isothermal, linear and self-rewetting cases are shown in Fig. 2 in the supplementary information, and the corresponding animations can be seen in Supplementary Videos 1, 2 and 3.

In Fig. 2(b), it can be seen that in the isothermal case, the value of $q / q_{0}$ remains 1 till $t \approx 13$ after which they repel each other. This repulsion behaviour of the bubbles observed in the isothermal case is consistent with the previous findings at low Reynolds numbers $[\underline{6}$. In contrast, in the linear and self-rewetting cases, the bubbles attract each other due to the Marangoni stresses. In the self-rewetting case, the rise velocities of the bubbles are quite low as compared to that in the case of the linear fluid, so they come closer slowly and touch each other at $t \approx 15$. Then, the bubbles display a repeated 'squeezing-relaxing' behaviour due to their shape deformations with a decreasing magnitude while they are in touch for $t>15$ (see Fig. 2(c)). In Fig. 2(c), it can also be seen that 
(a)

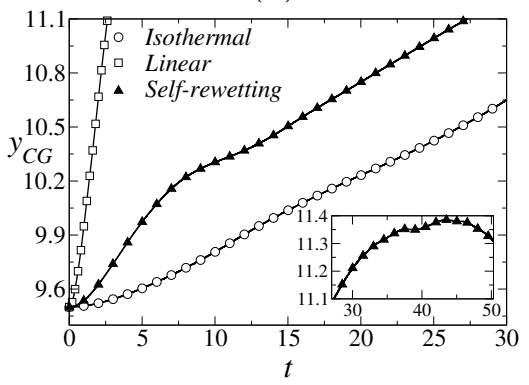

(b)

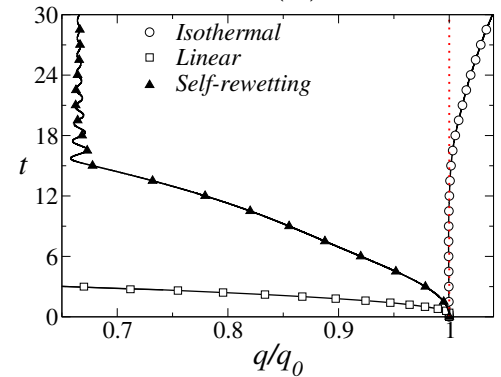

(c)

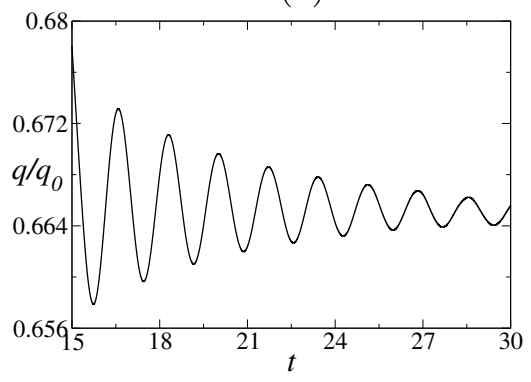

Figure 2: Temporal variations of (a) $y_{C G}$ of bubble 1 and (b) $q / q_{0}$ rising in isothermal, linear $\left(M_{1}=0.4, M_{2}=0\right)$ and self-rewetting $\left(M_{1}=0.4, M_{2}=0.2\right)$ fluids for $q_{0}=3$. (c) The oscillations observed for $t>15$ in the case of the self-rewetting fluid in panel (b). The rest of the parameter values are the same as the base parameters. The inset in panel (a) shows the late time behaviour for the self-rewetting case.

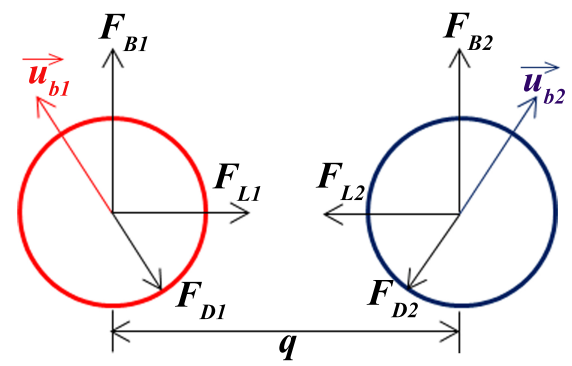

Figure 3: A free-body diagram showing the forces acting on the bubbles. Here, $F_{B 1}, F_{B 2}$, $F_{D 1}, F_{D 2}$ and $F_{L 1}, F_{L 2}$ are the buoyancy, viscous drag and lift forces acting on bubble 1 and bubble 2 , respectively. $\vec{u}_{b 1}$ and $\vec{u}_{b 2}$ are the velocities of bubbles 1 and 2, respectively. 
the average time period $\left(T_{p}\right)$ of the oscillations of $q / q_{0}$ is about 1.6. Note that, in the present study, $\mu_{A} / \beta_{1} \gamma$ is used as the time scale, which is associated with the Marangoni flow. In the present case, the value of the capillary time scale of the bubbles, $\sqrt{\rho_{A} R^{3} / \sigma}$ (or $\tau_{c}=\sqrt{W e /\left(1-M_{1} T+M_{2} T^{2}\right)}$ in the dimensionless form) is equal to 0.25 , which is calculated by assuming that $T=1$ at $y=y_{m}$ (near which the bubbles exhibit the oscillations). This infer that the observed oscillations are not of a capillary type, but rather driven by the Marangoni stresses. In the linear case, the bubbles do not display this behaviour as they rise faster due to the combined influence of the thermocapillary and buoyancy forces acting in the same direction. The distinct behaviours of the bubbles observed in the case of linear and self-rewetting fluids are also discussed below while analysing the forces acting on the bubbles.

Below we explore the repulsive and attractive behaviours of the bubbles by analysing the drag and lift forces acting on the bubbles and the flow field around the bubbles. A free body diagram showing the forces acting on the bubbles are depicted in Fig. 3 The drag and lift forces acting on the bubbles are calculated using the theoretical model derived by de Vries et al. 37] for a spherical bubble bouncing against a wall in a viscous liquid. Previously, Zhang et al. 38, also used a similar approach to estimate the force between two spherical bubbles rising side-by-side in an isothermal system. In a symmetrical situation, the magnitudes of $F_{B 1}=F_{B 2}=F_{B}, F_{D 1}=F_{D 2}=F_{D}$ and $F_{L 1}=F_{L 2}=F_{L}$. The dimensionless equations for the viscous drag $\left(F_{D}\right)$ and lift $\left(F_{L}\right)$ forces for two-dimensional bubbles rising side-by-side are given by

$$
\begin{aligned}
& F_{D}=\mathcal{V}\left(\frac{2}{F r}-\frac{d M_{y} v_{b}}{d t}\right), \\
& F_{L}=\mathcal{V}\left(\frac{d M_{x} u_{b}}{d t}-\frac{1}{2}\left[u_{b}^{2} \frac{d M_{x}}{d x}+v_{b}^{2} \frac{d M_{y}}{d x}\right]\right),
\end{aligned}
$$

where $\mathcal{V}$ is the dimensionless volume of the bubble; $u_{b}$ and $v_{b}$ are the horizontal and vertical components of the dimensionless bubble velocity. The dimensionless added mass coefficients, $M_{x}$ and $M_{y}$ are given by

$$
M_{x}=M_{y}=2+\left(\frac{1}{x_{b}}\right)^{2}
$$


(a)

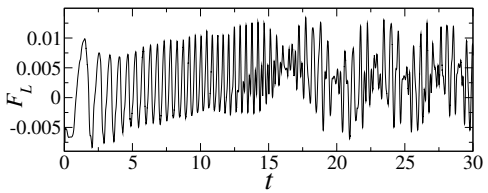

(c)

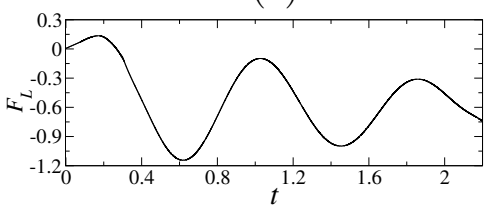

(e)

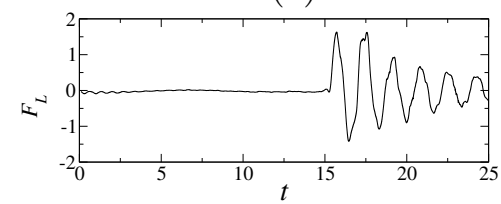

(b)

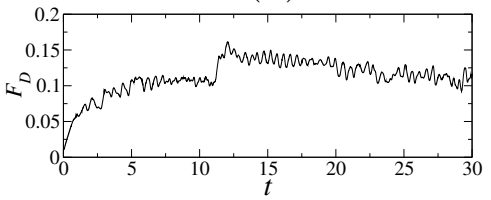

(d)

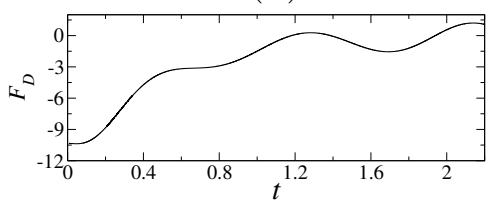

(f)

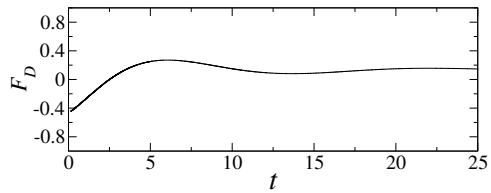

Figure 4: Temporal variations of the $(\mathrm{a}, \mathrm{c}, \mathrm{e})$ lift and $(\mathrm{b}, \mathrm{d}, \mathrm{f})$ viscous drag forces for $(\mathrm{a}, \mathrm{b})$ isothermal, $(\mathrm{c}, \mathrm{d})$ linear $\left(M_{1}=0.4, M_{2}=0\right)$ and $(\mathrm{e}, \mathrm{f})$ self-rewetting $\left(M_{1}=0.4, M_{2}=0.2\right)$ fluids. The rest of the parameter values are the same as those in Fig. 2

where $x_{b}$ is the distance of the bubble from the centreline of the channel $(x=0)$.

The lift and drag forces calculated from the numerically obtained results using Eqs. (8) and (9) have been plotted for one of the two bubbles (on the right-hand side) for the isothermal, linear and self-rewetting cases in Fig. 4 It is evident from Fig. 4(a) that the lift force is negligible for bubbles in the isothermal system. In contrast to this, there is a cyclic attractive force (i.e. $\left.F_{L}<0\right)$ on the bubbles in the system where the surface tension varies linearly with temperature (see Fig. 4(c)). The self-rewetting case shows negligible lift till $t \approx 15$ and then displays a cyclic attractive-repulsive force which diminishes in magnitude with time, as shown in Fig. 4(e). The initial dynamics for $t<15$ showing negligible lift may be attributed to a lower magnitude of the surface tension gradients in the vicinity of the bubbles. For $t>15$, while the bubbles are in touch, the lift force acting between the bubbles oscillates about zero as evident in Fig. 4e with a time period of about 1.7. It can be seen that the 
time period of the oscillations of the lift force for $t>15$ is the same as the time period of the variation of $q / q_{0}$ as shown in Fig. 22. Thus, the bubbles exhibit a squeezing and relaxing behaviour until the oscillations in the lift force die down due to the viscous force. This also indicates that Marangoni flow causes the oscillations seen in Figs. 2(b,c) as the bubbles come into contact in the self-rewetting fluid, since the lift force is a consequence of the Marangoni flow. The drag force on bubbles in the isothermal system (Fig. 4(b)) reaches a positive quasi-steady value at larger times; however, the drag force on the linear and self-rewetting systems approach a negligibly small value (Figs. 4(d) and (f)) as the time progresses. This is due to a temporary balance between thermocapillary and buoyancy forces in the case of the linear and self-rewetting systems.

More insights into the dynamics of the isothermal, linear and self-rewetting systems can be gained by visualising the flow in and around the bubbles as they rise. Fig. 5 shows the streamlines (in the frame of reference of the rising bubbles) and temperature contours in these systems for $q_{0}=3$ at different time instants. In the isothermal system (Fig. 5(a)), it can be noticed that the bubbles are gradually departing away from each other. Bubbles in the linear system (Fig. 5(b)) tend to attract each other and the vortical flow inside the bubbles suggests a tendency of the bubbles to come closer to each other. [17. also analysed the interaction of the streamline patterns in the case of two bubbles rising in a linear fluid. It can also be seen that, in the linear case, the bubbles deform to slightly tilted oblate shapes due to the combined action of buoyancy and thermocapillary forces. In this case, as the temperature of the air inside the bubbles increases slowly, the colder regions at the centre of the bubbles are clearly visible at $t=0.8$ in Fig. 5. As the time progresses, this colder region becomes lighter and rises up to form a ring at the upper part of the bubbles. The thermocapillary force resulting due to the surface tension gradient can be resolved in the horizontal and vertical directions. In the case of linear fluid, while the horizontal component contributes to the lift force, the vertical component acts in the upward direction (in the same direction as that of the 
(a)
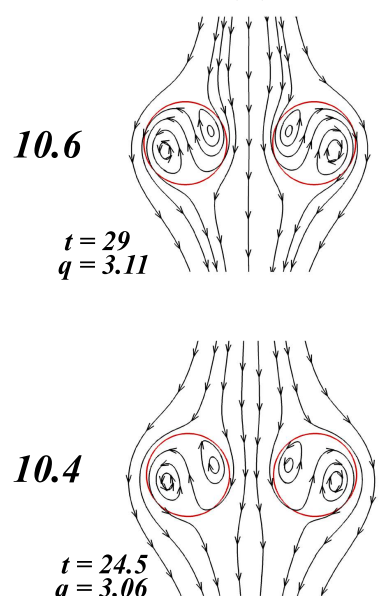

$q=3.06$
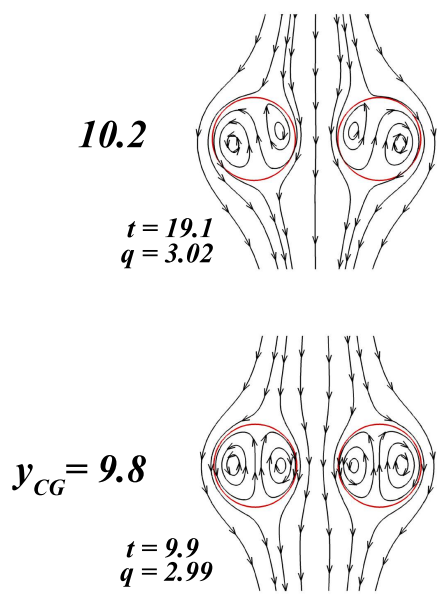

(b)
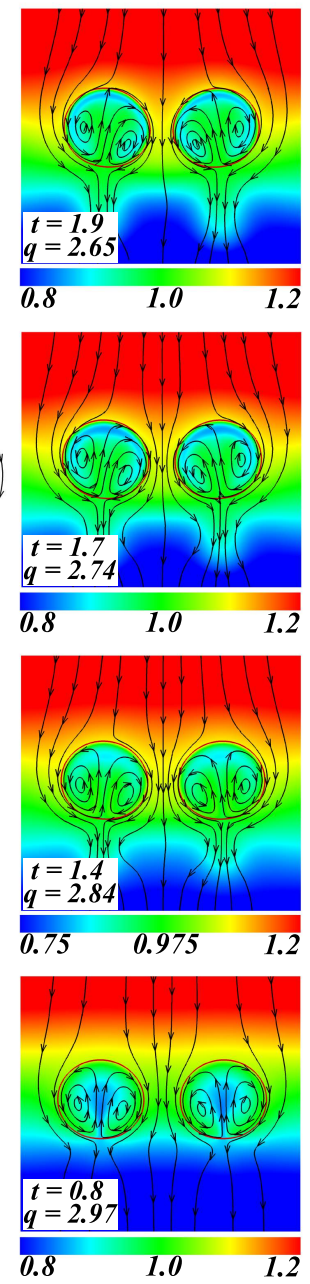

(c)
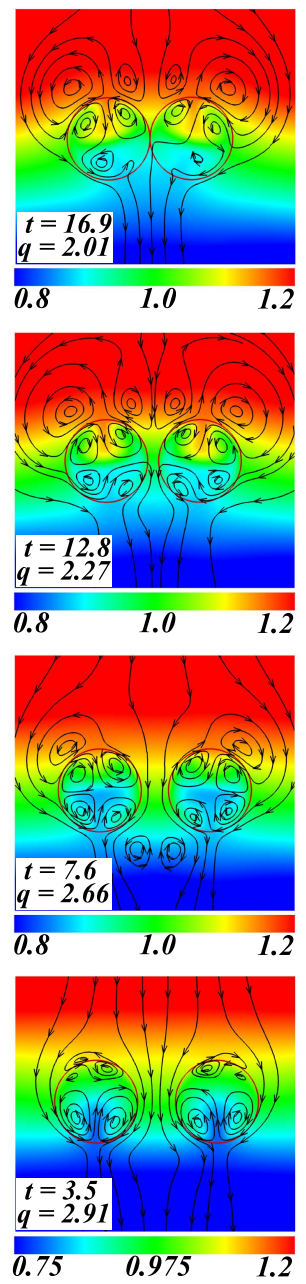

Figure 5: Streamlines at different times for two bubbles rising in (a) isothermal, (b) linear $\left(M_{1}=0.4, M_{2}=0\right)$ and (c) self-rewetting $\left(M_{1}=0.4, M_{2}=0.2\right)$ fluids for $q_{0}=3$. The streamlines are plotted in the frame of reference of the rising bubbles. The color contour in panels (b) and (c) represents the temperature field. 
buoyancy force). In contrast, in the case of the self-rewetting fluid, after the bubbles cross the location of minimum surface tension $\left(y_{m}=10\right.$, see Fig. 1 (b)), the surface tension increases in the vertical direction (opposite to that in the case of a linear fluid). Thus, the bubbles experience a downward force (opposing the buoyancy force), in addition to the horizontal oscillatory lift force, due to the thermo-capillary stresses. Thus, in case of the self-rewetting fluid (Fig. 5(c)), the bubbles come in contact and display repeated squeezing and relaxing behaviour at later times as discussed above. Another interesting phenomenon can also be observed in the self-rewetting fluid. It can be seen that at early times (see, for instance, $t=3.5$ ) a colder plume enters from the bottom part of each bubble, which diffuses inside the bubble (see $t=7.6$ ). At this stage a hotter plume enters from the top part of each bubble and grows as the time progresses. The fingers of the cold and hot plumes set a temperature gradient across the interface, resulting in two sets of clockwise and counter-clockwise rotating vortical regions, unlike the isothermal and linear cases.

Further, we analyse the orientation of one of the bubbles (bubble 1) as a function of time as they rise in Figs. 6(a), (b) and (c) for the isothermal, linear and self-rewetting cases, respectively. The orientation of the bubble 1 can be defined as $\tan ^{-1}\left(u_{x} / u_{y}\right)$, wherein $u_{x}$ and $u_{y}$ are the $x$ and $y$ components of the velocity of the centre of gravity of the bubble. So while a positive value of the orientation indicates that the bubbles approach each other, a negative value demonstrates repulsion. It can be seen that in the isothermal case (Fig. 6 (a)), the bubbles exhibit an initial attraction for a short time and then gradually move apart. In contrast, in the linear and self-rewetting cases, the bubbles show attractive behaviour till the point of collision (the dynamics of the bubbles after the collision will be discussed below). The bubbles approach each other more rapidly in the case of the linear fluid as compared to that in the case of the self-rewetting fluid. In the self-rewetting fluid, it can be observed that the orientation of the bubble undergoes oscillations whose amplitude decreases with time (Fig. 6(c)). These oscillations are also related to the oscillations observed in Fig. 2(c). Figs. 7(a) and (b) show the surface tension contours along the 
(a)

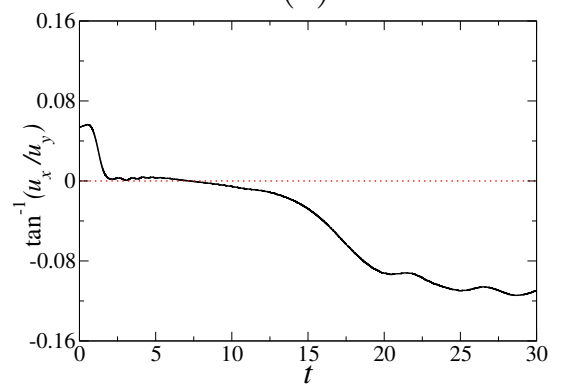

(b)

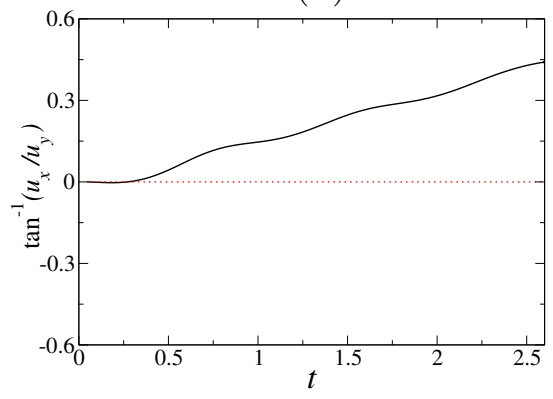

(c)

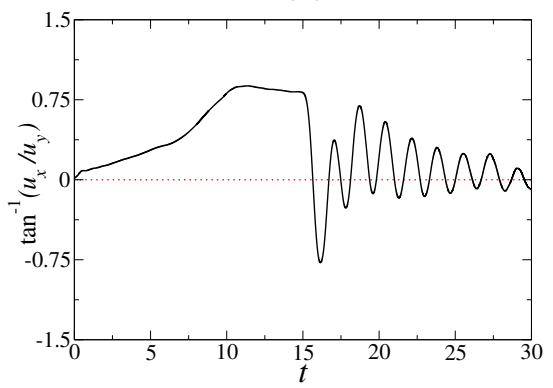

Figure 6: Temporal variations of orientation of bubble $1\left(\tan ^{-1}\left(u_{x} / u_{y}\right)\right)$ in (a) isothermal medium, (b) linear fluid $\left(M_{1}=0.4, M_{2}=0\right)$ and (c) self-rewetting fluid $\left(M_{1}=0.4, M_{2}=0.2\right)$ for $q_{0}=3$. The rest of the parameter values are the same as the base parameters.

interface of the bubbles rising in the linear and self-rewetting fluids, respectively. Note that the interface has been made thicker for better visualisation. As expected, in the case of the linear fluid, the bubbles show a high surface tension region at the bottom part and a low surface tension region at the top part of the bubbles as the temperature increases as we move in the vertical direction (Fig. 7(a)). This changes the orientation of the vortical flow (two recirculating regions) inside the bubbles observed in the isothermal system (see Fig. 5). On the other hand, dramatically different vortical flow patterns are observed in the case of the self-rewetting fluid due to the non-monotonic surface tension profile along the interface separating the fluids (Fig. 7(b)), which induces two sets of clockwise and counterwise rotating vortical regions.

In order to understand the nature of collision of the bubbles in linear and 
(a)

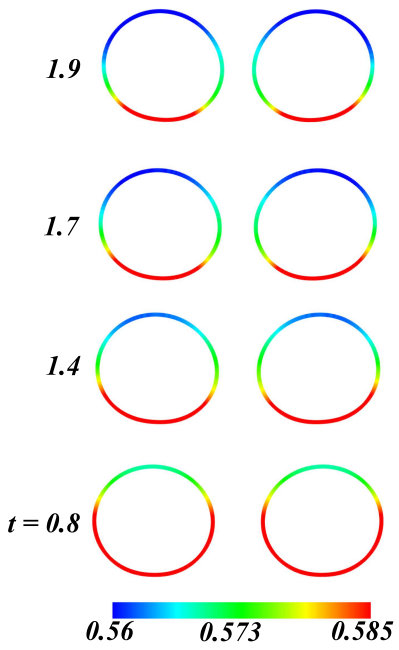

(b)

16.9

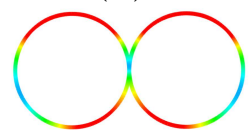

12.8

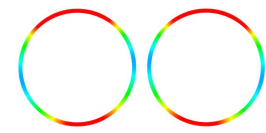

7.6
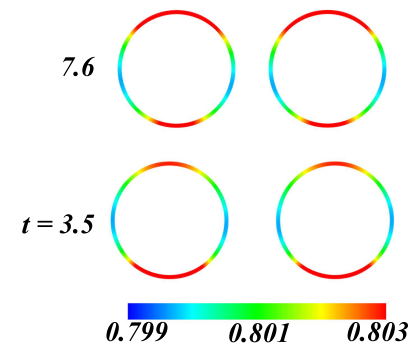

Figure 7: Variation of the surface tension along the interface (widened for visibility) of the bubbles rising in the (a) linear fluid $\left(M_{1}=0.4, M_{2}=0\right)$ and (b) self-rewetting fluid $\left(M_{1}=0.4\right.$, $\left.M_{2}=0.2\right)$ for $q_{0}=3$. The rest of the parameter values are the same as the base parameters.

self-rewetting fluids, the temporal variations of $y_{C G}$ of bubble 1 rising in a linear $\left(M_{1}=0.4, M_{2}=0\right)$ and self-rewetting $\left(M_{1}=0.4, M_{2}=0.2\right)$ fluids, have been plotted in Figs. 8(a) and (b). Here $q_{0}=2.5$, and the rest of the parameters are the same as the base parameters. It can be seen in Fig. 8 (a) that the bubbles undergo large deformation and come in contact at $t \approx 1.5$. The bubbles remain in contact till $t=2.6$ while they continue to deform. Subsequently, they repel and move away from each other at later times $(t>2.6)$. In contrast, in the case of the self-rewetting fluid, it can be seen in Fig. 8(b) that the bubbles experience an attractive lift force and they collide at $t \approx 9$. The bubbles remain in contact hereafter and exhibit a repeated squeezing-relaxing behaviour as they undergo small-amplitude oscillations. In this case, the bubbles are mostly spherical at all instants. So it can be inferred that in the self-rewetting case the collision is plastic in nature, while in the case of linear fluid it is not.

The effect of the initial separation distance between the bubbles, $q_{0}$ is studied in Fig. 9. As expected, it can be seen that the intermediate decelerating 
(a)

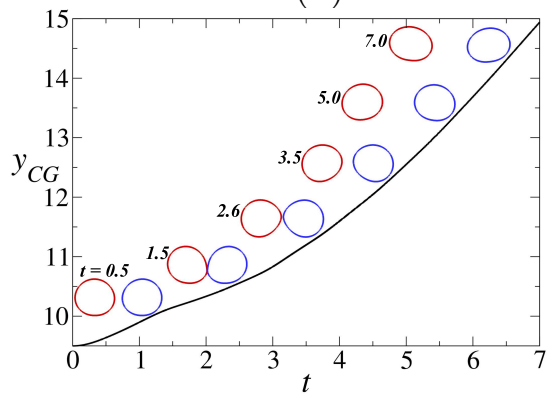

(b)

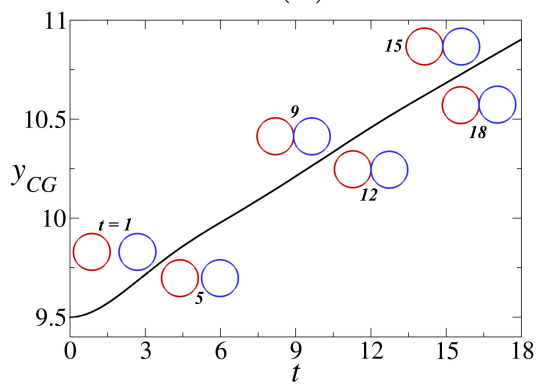

Figure 8: Temporal variations of $y_{C G}$ of the bubbles rising in a (a) linear fluid $\left(M_{1}=0.4\right.$, $\left.M_{2}=0\right)$ and (b) self-rewetting fluid $\left(M_{1}=0.4, M_{2}=0.2\right)$ for $q_{0}=2.5$. The rest of the parameters are the same as the base parameters. The corresponding shapes of the bubbles are shown at different time instants.

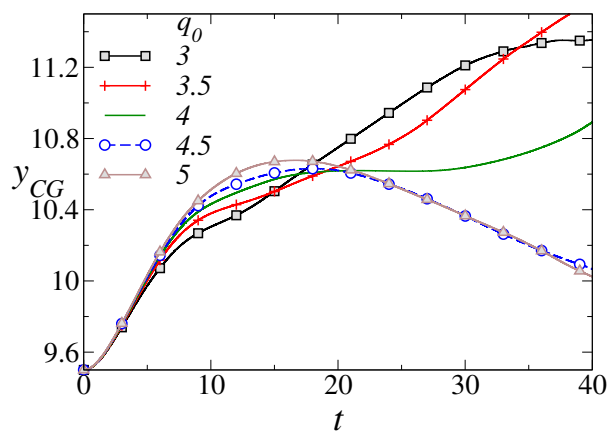

Figure 9: Temporal variations of $y_{C G}$ of bubble 1 in a self-rewetting fluid $\left(M_{1}=0.4, M_{2}=0.2\right)$ for different values of $q_{0}$. The rest of the parameter values are the same as the base parameters. 


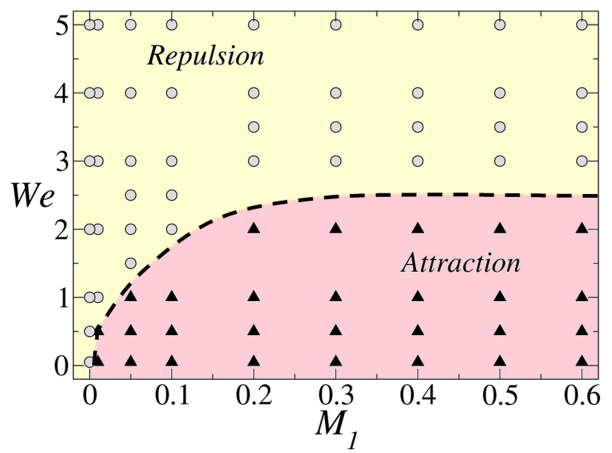

Figure 10: Regime map demarcating the attractive and repulsive bubbles in $W e-M_{1}$ space for $q_{0}=3$ and $M_{2}=M_{1} / 2$. The filled circle and triangle symbols represent the repulsive and attractive bubbles, respectively. The rest of the parameter values are the same as the base parameters.

phase increases with the increase in $q_{0}$ as the interaction between the bubbles decreases. For $q_{0} \geq 4.5$, the behaviours of the bubbles are almost independent of each other. For $q_{0} \geq 4.5$, the bubbles exhibit motion reversal after crossing the location of the minimum surface tension, which is similar to that observed in the case of a single gas bubble rising in a non-isothermal self-rewetting fluid [26.

As discussed above the attraction of the bubbles observed at a low Reynolds number in a non-isothermal self-rewetting system (in contrast to the repulsive behaviour in an isothermal system) is due to the thermocapillary effect, we plot a regime map demarcating the attractive and repulsive behaviours in $W e-M_{1}$ space for $q_{0}=3$ in Fig. 10. It can be seen that the bubbles exhibits repulsive motion for all values of $W e$ for $M_{1}=0$ (isothermal case). Increasing the value of $M_{1}$ increases the critical value of the Weber number at which the transition between the attractive and repulsive behaviours takes place. The critical Weber number achieves a plateau of about 2.5 for $M_{1} \geq 0.3$ for the base parameters considered in this study. Figs. 11(a,c) and (b,d) depict the temporal variations of $q / q_{0}$ for different values of $W e$ for $M_{1}=0.4$ and different values of $M_{1}$ for $W e=1$, respectively. Here, panels $(\mathrm{a}, \mathrm{b})$ and $(\mathrm{c}, \mathrm{d})$ correspond to self-rewetting 
(a)

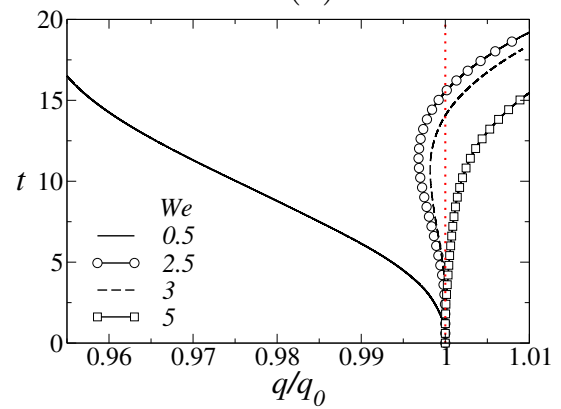

(c)

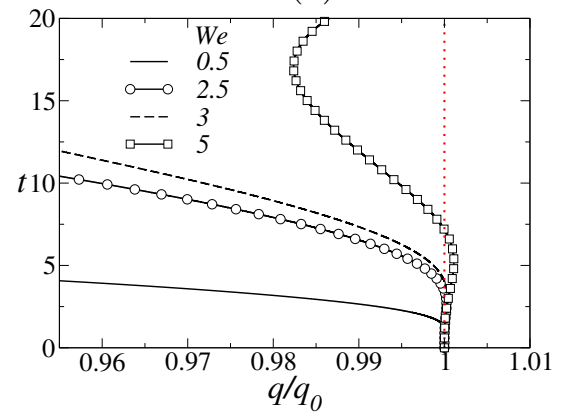

(b)

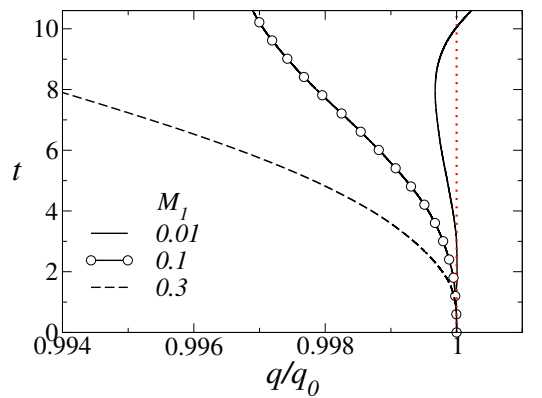

(d)

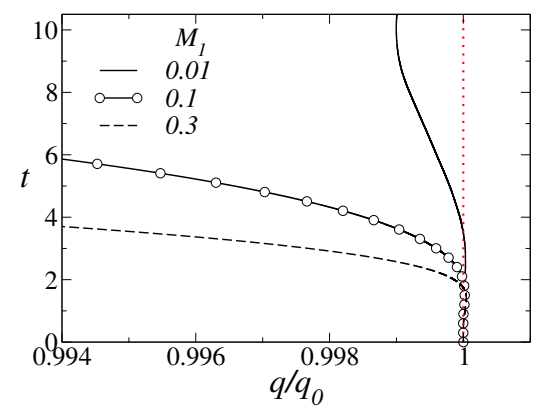

Figure 11: (a,c) Effect of Weber number on the temporal variations of $q / q_{0}$ : (a) $M_{1}=0.4$ $M_{2}=0.2$ (a self-rewetting fluid) and (c) $M_{1}=0.4, M_{2}=0$ (a linear fluid). (b,d) Effect of $M_{1}$ on the temporal variations of $q / q_{0}$ for $W e=1$ : (b) $M_{2}=M_{1} / 2$ (self-rewetting fluids) and (d) $M_{2}=0$ (linear fluids). The rest of the parameter values are the same as the base parameters.

(a)

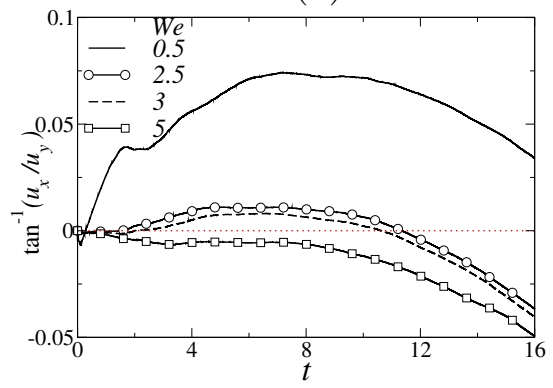

(b)

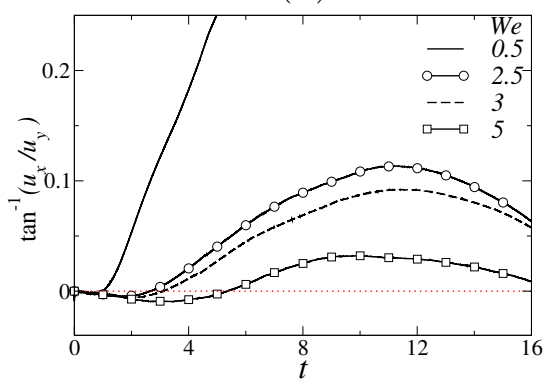

Figure 12: Temporal variations of orientation of bubble $1\left(\tan ^{-1}\left(u_{x} / u_{y}\right)\right)$ in (a) $M_{1}=0.4$ $M_{2}=0.2$ (a self-rewetting fluid) and (c) $M_{1}=0.4, M_{2}=0$ (a linear fluid). The rest of the parameter values are the same as those used to generate Fig. 11 
and linear fluids, respectively. For self-rewetting fluid, it can be seen in Fig. 11(a) that while the bubbles clearly exhibit an attractive motion for $W e=0.5$, and they repel each other after rising in nearly straight paths $\left(q / q_{0} \approx 1\right)$ at the early times for $W e \geq 2.5$. In contrast, in the case of linear fluid, the repulsive behaviour is observed only for $W e=5$. It is to be noted here that the bubbles in the linear fluid undergo large deformation as shown in Fig. 8(a), whereas the bubbles remain spherical even for $W e=5$ in the case of self-rewetting fluid. This indicates that the deformation increases the interaction of the boundary layers around the bubbles, which in turn enhances the attractive motion. The influence of the Weber number on the orientation of the bubble 1 for self-rewetting and linear fluids is presented in Figs. 12(a) and (b), respectively. It can be seen that the value of $\tan ^{-1}\left(u_{x} / u_{y}\right)$ in the linear fluid is much large than that of the self-rewetting fluid which suggests that the bubbles in the linear fluid are more attractive than the self rewetting fluid. It is also observed (not shown) that increasing $W e$ decreases the lift force between the bubbles. Inspection of Figs. 11(b) and (d) also reveals that the tendency of the attractive motion is more in the case of linear fluid as compared to the self-rewetting fluid for each value of $M_{1}$ (i.e., the rate of decrease of $q / q_{0}$ is more in linear fluid as compared to the self-rewetting fluid). However, in the case of linear fluid, after the collision, the bubbles move away from each other while in the self-rewetting fluid they stay in contact as shown in Fig. 8

\section{Concluding remarks}

The interaction of two non-coalescing gas bubbles rising side-by-side in a non-isothermal self-rewetting fluid is investigated. The rise dynamics of two bubbles at low Reynolds number was shown to be planar by Tripathi et al. [7, thus a two-dimensional geometry is considered in the present study. It is well known that two side-by-side bubbles with a separation distance below a critical value in an isothermal medium are separated as they rise at low Reynolds numbers [6]. On the other hand, we found that in a non-isothermal system with 
linear and self-rewetting fluids, two gas bubbles attract each other for the same set of parameters that lead to a repulsive motion in an isothermal medium. In the case of the self-rewetting fluid, the bubbles exhibit a squeezing-relaxing behaviour after crossing the location of the minimum surface tension due to the thermocapillary stresses. Unlike the bubbles rising in the linear fluid, where the bubbles attract and then bounce after the collision, it is found that the collision between the bubbles in the self-rewetting fluid is plastic in nature. The attractive and repulsive behaviours of the gas bubbles are found to be a function of the Weber number $(W e)$ and the linear component of the surface tension gradient with temperature $\left(M_{1}\right)$. However, it is observed that while the air bubbles deformed significantly in the case of the linear fluid, they remain spherical in the self-rewetting fluid even for the highest Weber number considered in the present study. This indicates that the attractive behaviour of the bubbles in the case of the self-rewetting fluid is due to the lift force produced by the thermocapillary stresses and not due to the deformation. A regime map demarcating the repulsive and attractive behaviours in $W e-M_{1}$ space reveals that the bubbles in a non-isothermal self-rewetting fluid experience attractive motion in the low $W e$ and high $M_{1}$ region and the critical value of $W e$ increases with increasing $M_{1}$ and obtains a plateau value of about 2.5 for $M_{1} \geq 0.3$ for the parameters considered in the present study. The mechanism underlying the observed phenomena is elucidated by examining the drag-and-lift forces acting on the bubbles, the streamlines and the temperature field and orientations of the bubbles.

Declaration of interests: The authors report no conflict of interest.

Acknowledgement: K. C. S. thanks Science and Engineering Research Board, India for providing financial support through the grant number, MTR/2017/000029. 


\section{References}

[1] N. Kantarci, F. Borak, K. O. Ulgen, Bubble column reactors, Process Biochem 40 (7) (2005) 2263-2283.

[2] B. Aboulhasanzadeh, G. Tryggvason, Effect of bubble interactions on mass transfer in bubbly flow, Int. J. Heat Mass Transfer. 79 (2014) 390-396.

[3] D. C. Blanchard, Comments on the breakup of raindrops, J. Atmos. Sci. 19 (1962) 119-120.

[4] D. C. Blanchard, The electrification of the atmosphere by particles from bubbles in the sea, Prog.Oceanogr. 1 (1963) 73-202.

[5] J. B. W. Kok, Dynamics of a pair of gas bubbles moving through liquid. Part I. Theory., Eur. J. Mech. B/Fluids 12 (1993) 515-540.

[6] D. Legendre, J. Magnaudet, G. Mougin, Hydrodynamic interactions between two spherical bubbles rising side by side in a viscous liquid, J. Fluid Mech. 497 (2003) 133-166.

[7] M. K. Tripathi, A. R. Premlata, K. C. Sahu, R. Govindarajan, Two initially spherical bubbles rising in quiescent liquid, Phys. Rev. Fluids 2 (7) (2017) 073601 .

[8] M. Balla, S. Kavuri, M. K. Tripathi, K. C. Sahu, R. Govindarajan, Effect of viscosity and density ratios on two drops rising side by side, Phys. Rev. Fluids 5 (1) (2020) 013601.

[9] R. S. Subramanian, R. Balasubramaniam, G. Wozniak, Fluid mechanics of bubbles and drops, in: R. Monti (Ed.), Physics of Fluids in Microgravity, Taylor and Francis, London, 149-177, 2002.

[10] S. Nas, G. Tryggvason, Thermocapillary interaction of two bubbles or drops, Int. J. Multiphase Flow 29 (7) (2003) 1117-1135. 
[11] S. Nas, M. Muradoglu, G. Tryggvason, Pattern formation of drops in thermocapillary migration, Int. J. Heat Mass Transfer. 49 (2006) 2265-2276.

[12] N. O. Young, J. S. Goldstein, M. J. Block, The motion of bubbles in a vertical temperature gradient, J. Fluid Mech. 6 (1959) 350-356.

[13] L. Zhang, R. S. Subramanian, R. Balasubramaniam, Motion of a drop in a vertical temperature gradient at small Marangoni number - the critical role of inertia, J. Fluid Mech. 448 (2001) 197-211.

[14] H. Liu, Y. Zhang, A. J. Valocchi, Modeling and simulation of thermocapillary flows using lattice Boltzmann method, J. Comput. Phys. 231 (12) (2012) 4433-4453.

[15] I. Seric, S. Afkhami, L. Kondic, Direct numerical simulation of variable surface tension flows using a Volume-of-Fluid method, J. Comput. Phys. 352 (1) (2018) 615-636.

[16] M. K. Tripathi, K. C. Sahu, Motion of an air bubble under the action of thermocapillary and buoyancy forces, Computers \& Fluids 177 (2018) $58-68$.

[17] H. Wei, R. S. Subramanian, Migration of a pair of bubbles under the combined action of gravity and thermocapillarity, J. Colloid Interface Sci. 172 (2) (1995) 395-406.

[18] O. M. Lavrenteva, V. Berejnov, A. Nir, Axisymmetric motion of a pair of deformable heavy drops in an upward temperature gradient, J. Colloid Interface Sci. 255 (1) (2002) 214-217.

[19] O. Frolovskaya, A. Nir, O. M. Lavrenteva, Stationary regimes of axisymmetric thermal wake interaction of two buoyant drops at low Reynolds and high Peclet number, Phys. Fluids 18 (7) (2006) 072103.

[20] R. Vochten, G. Petre, Study of heat of reversible adsorption at air-solution interface 2. Experimental determination of heat of reversible adsorption of some alcohols, J. Colloid Interface Sci. 42 (1973) 320-327. 
[21] G. Petre, M. A. Azouni, Experimental evidence for the minimum of surface tension with temperature at aqueous alcohol solution air interfaces, J. Colloid Interface Sci. 98 (1984) 261-263.

[22] M. C. Limbourgfontaine, G. Petre, J. C. Legros, Thermocapillary movements under at a minimum of surface tension, Naturwissenschaften 73 (1986) 360-362.

[23] Y. Abe, A. Iwasaki, K. Tanaka, Microgravity experiments on phase change of self-rewetting fluids, Ann. N.Y. Acad. Sci. 1027 (2004) 269-285.

[24] G. Karapetsas, K. C. Sahu, K. Sefiane, O. K. Matar, Thermocapillarydriven motion of a sessile drop: effect of non-monotonic dependence of surface tension on temperature, Langmuir 30 (15) (2014) 4310-4321.

[25] K. Chaudhury, S. Chakraborty, Spreading of a droplet over a nonisothermal substrate: Multiple scaling regimes, Langmuir 31 (14) (2015) 4169-4175.

[26] M. K. Tripathi, K. C. Sahu, G. Karapetsas, K. Sefiane, O. K. Matar, Nonisothermal bubble rise: non-monotonic dependence of surface tension on temperature, J. Fluid Mech. 763 (2015) 82-108.

[27] M. Balla, M. K. Tripathi, K. C. Sahu, G. Karapetsas, O. K. Matar, Nonisothermal bubble rise dynamics in a self-rewetting fluid: three-dimensional effects, J. Fluid Mech. 858 (2019) 689-713.

[28] B. R. Duffy, S. K. Wilson, J. J. A. Conn, K. Sefiane, Unsteady motion of a long bubble or droplet in a self-rewetting system, Phys. Rev. Fluids 3 (12) (2018) 123603.

[29] F. Blanchette, A. M. Shapiro, Drops settling in sharp stratification with and without Marangoni effects, Phys. Fluids 24 (4) (2012) 042104.

[30] A. Doostmohammadi, S. Dabiri, A. M. Ardekani, A numerical study of the dynamics of a particle settling at moderate Reynolds numbers in a linearly stratified fluid, J. Fluid Mech. 750 (2014) 5-32. 
[31] Y. Li, C. Diddens, A. Prosperetti, K. L. Chong, X. Zhang, D. Lohse, Bouncing oil droplet in a stratified liquid and its sudden death, Phys. Rev. Lett. $122(15)(2019) 154502$.

[32] R. Savino, A. Cecere, S. Van Vaerenbergh, Y. Abe, G. Pizzirusso, W. Tzevelecos, M. Mojahed, Q. Galand, Some experimental progresses in the study of self-rewetting fluids for the SELENE experiment to be carried in the Thermal Platform 1 hardware, Acta Astronautica 89 (2013) 179-188.

[33] G. D. Weymouth, D. K. P. Yue, Conservative Volume-of-Fluid method for free-surface simulations on Cartesian-grids, J. Comput. Phys. 229 (2010) 2853-2865.

[34] S. Popinet, Gerris: a tree-based adaptive solver for the incompressible Euler equations in complex geometries, J. Comput. Phys. 190 (2003) 572-600.

[35] S. Popinet, An accurate adaptive solver for surface-tension-driven interfacial flows, J. Comput. Phys. 228 (2009) 5838-5866.

[36] D. Mamalis, V. Koutsos, K. Sefiane, Nonisothermal spreading dynamics of self-rewetting droplets, Langmuir 34 (5) (2018) 1916-1931.

[37] A. W. G. de Vries, A. Biesheuvel, L. Van Wijngaarden, Notes on the path and wake of a gas bubble rising in pure water, Int. J. Multiphase Flow 28 (11) (2002) 1823-1835.

[38] J. Zhang, L. Chen, M. J. Ni, Vortex interactions between a pair of bubbles rising side by side in ordinary viscous liquids, Phys. Rev. Fluids 4 (4) (2019) 043604. 